\title{
Nucleon spin and quark content at the physical point
}

\section{Constantia Alexandrou*}

Department of Physics, University of Cyprus, CY-1678 Nicosia, Cyprus and Computation-based Science and Technology Research Center, The Cyprus Institute, 20, K. Kavafi Str., 2121 Nicosia, Cyprus

E-mail: alexandeucy.ac.cy

\section{Martha Constantinou, Kyriakos Hadjiyiannakou, Christos Kallidonis, Giannis Koutsou \\ Computation-based Science and Technology Research Center, The Cyprus Institute, 20, K. \\ Kavafi Str., 2121 Nicosia, Cyprus \\ E-mail: marthacetemple.edu, \\ k.hadjiyiannakou@cyi.ac.cy,c.kallidonis@cyi.ac.cy,g•koutsou@cyi.ac.cy}

\section{Karl Jansen, Christian Wiese}

NIC, DESY, Platanenallee 6, D-15738 Zeuthen, Germany

E-mail: karl.jansen@desy.de

\section{Alejandro Vaquero Avilés-Casco}

INFN Sezione di Milano-Bicocca, Edificio U2, Piazza della Scienza 3, 20126 Milano, Italy

E-mail: Alejandro.Vaquero@mib.infn.it

We present results on the spin and quark content of the nucleon using $N_{f}=2$ twisted mass cloverimproved fermion simulations with a pion mass close to its physical value. We use recently developed methods to obtain accurate results for both connected and disconnected contributions. We provide results for the axial charge, quark and gluon momentum fraction as well as the light, strange and charm $\sigma$-terms.

34th annual International Symposium on Lattice Field Theory

24-30 July 2016

University of Southampton, UK

\footnotetext{
* Speaker.
} 


\section{Introduction}

The contribution of quarks and gluons to the spin of the nucleon has being studied experimentally since a number of years. Measurements indicate that quarks contribute only about half to the spin of the nucleon. Although the quark contribution to the spin of the nucleon can be computed using lattice QCD from first principles, it is only very recently that such a calculation has become possible, without any approximations and directly at the physical value of the pion mass. We present our recent results on the intrinsic spin $\Delta \Sigma^{q}$ and total spin $J^{q}$ taking into account disconnected contributions, which at the physical point cannot be neglected.

Another important quantity is the quark contents of the nucleon given by $\sigma_{f} \equiv m_{f}\left\langle N\left|\bar{q}_{f} q_{f}\right| N\right\rangle$, where $q_{f}$ is a quark field of flavor $f$ and $|N\rangle$ is the nucleon state. Beyond probing chiral symmetry breaking, a precise evaluation of these quantities will reduce the uncertainty in interpreting experiments for dark matter searches since the Higgs-nucleon coupling depends on $\sigma_{f}$ [1]. We present here a direct evaluation of the light quark $\sigma_{\pi N}$, the strange quark $\sigma_{s}$ and charmed quark $\sigma_{c}$ contents of the nucleon. In this direct evaluation one computes the three-point function of the scalar operator at zero momentum transfer, which additionally yields the scalar charge after appropriate renormalization. Since charges are the simplest matrix elements to evaluate, we start by discussing our results on the scalar and tensor charges, as well as the axial charge which yields the contribution of intrinsic quark spins to the nucleon spin. The results are based on an ensemble of $N_{f}=2$ twisted mass fermions with clover improvement, on a lattice size of $48^{3} \times 96$, lattice spacing $a=$ 0.093(1) fm and pion mass $m_{\pi}=131 \mathrm{MeV}$, referred to hereon as the physical ensemble [2, 3, 4].

\section{Nucleon charges: $g_{A}, g_{s}, g_{T}$}

We consider the nucleon matrix elements $\left.\left\langle N\left(\vec{p}^{\prime}\right) \mathscr{O}_{X} N(\vec{p})\right\rangle\right|_{q^{2}=0}$ of the scalar operator, $\mathscr{O}_{S}^{a}=$ $\bar{\psi}(x) \frac{\tau^{a}}{2} \psi(x)$ the axial-vector operator, $\mathscr{O}_{A}^{a}=\bar{\psi}(x) \gamma^{\mu} \gamma_{5} \frac{\tau^{a}}{2} \psi(x)$ and the tensor operator $\mathscr{O}_{T}^{a}=\bar{\psi}(x) \sigma^{\mu \nu} \frac{\tau^{a}}{2} \psi(x)$ The isovector combination, given by taking the Pauli matrix $\tau^{3}$, has no disconnected contributions and we therefore first discuss the isovector charges. While the axial charge is well-known experimentally, $g_{T}$ and $g_{S}$ are not, however there is an experimental program at JLab to improve the accuracy on the value of $g_{T}$.
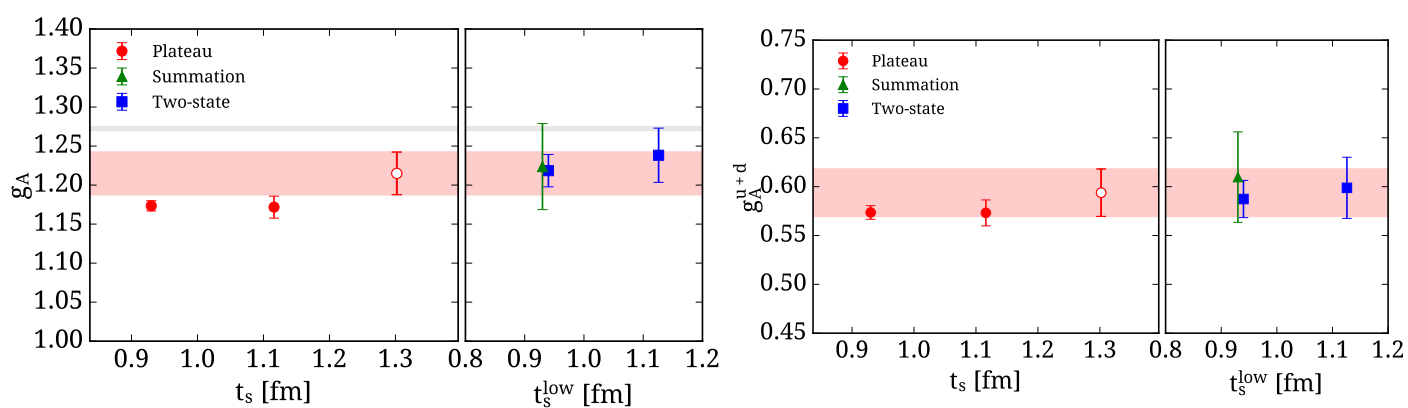

Figure 1: Isovector (left) and isoscalar connected (right) axial charge for three sink-source time separations: $t_{s} / a=10,12,14$, using 9260 measurements. $t_{s}^{\text {low }}$ is the lowest value of $t_{s}$ used in the fits. The grey band is the experimental value. The open symbol is the value we take.

In Fig. 1 we show the value of the isovector and isoscalar axial charge as we change the sinksource time separation $t_{s}$ form $0.93 \mathrm{fm}$ to $1.3 \mathrm{fm}$, as well as, the results when using the summation 
method and a fit including the first excited state (two-state fit). For $t_{S} / a=14$ there is an increase in the value of $g_{A}$, which however remains below the experimental value while the two-state fit using $t_{s} / a=12$ and $t_{s} / a=14$ and the summation method both having a larger error yielding consistent values. The connected contribution to the isoscalar axial charge shows convergence, with results from all methods being in agreement. We perform a similar analysis for $g_{S}^{u-d}$ and $g_{T}^{u-d}$, where for the scalar we increase $t_{s}$ to $1.7 \mathrm{fm}$ in order to sufficiently suppress excited states. We used statistics of $\sim 9260$ for $t_{s} / a=10,12$, and $14, \sim 48000$ for $t_{s} / a=16$ and $\sim 70000$ for $t_{s} / a=18$ to keep the errors approximately constant. The values are listed in Table I.
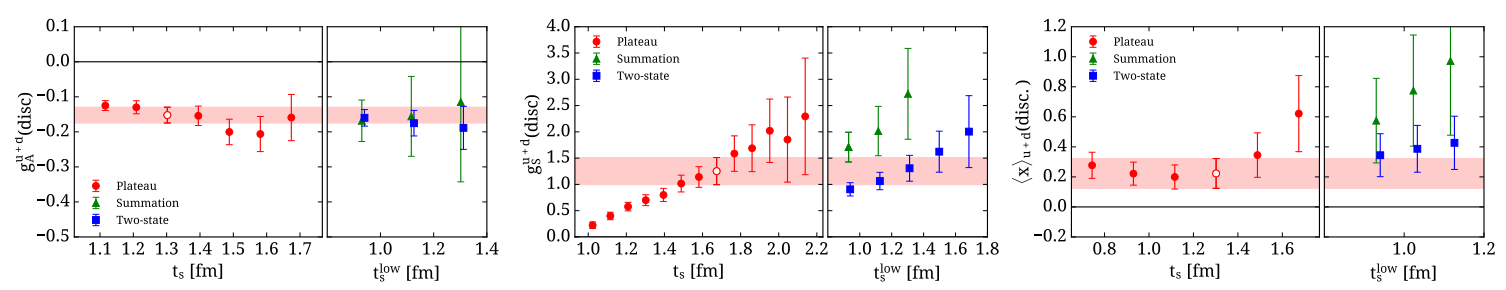

Figure 2: Disconnected contributions to the isoscalar axial charge (left), to the scalar charge (middle) and to the momentum fraction (right) shown as a function of $t_{s}$.

Besides the connected contributions, isoscalar nucleon charges also receive disconnected contributions. Combining deflation of the low-lying eigenvalues and the truncated solver method (TSM) we are able to compute these quantities to sufficient accuracy for the first time at the physical point [5]. GPUs are used to increase statistics with 100 source positions computed per gauge configuration for which we have checked that the error preserves the proper scaling. In Fig. 2 we show the disconnected contributions to $g_{A}^{u+d}$ and $g_{S}^{u+d}$ as we vary $t_{s}$ as well as the values from the summation method and the two-state fit. As expected, for the scalar one needs to increase $t_{s}$ in order to suppress excited states sufficiently to isolate the nucleon matrix element. In both the scalar and axial-vector the disconnected contribution are non-zero and need to be taken into account. Disconnected contributions to the tensor charge are consistent with zero. The statistics used for the evaluation of these disconnected contributions are approximately 850,000.

Nucleon $\sigma$-terms are computed either via the Feynman-Hellmann theorem using $\sigma_{q}=m_{l} \frac{\partial m_{N}}{\partial m_{q}}$ or by a direct computation of the scalar matrix element. We have computed the light, strange and charm $\sigma$-terms using our physical ensemble [6] by computing the nucleon matrix element of the scalar operator as shown in Fig. 2 for the connected contribution and in Fig. 3 for the disconnected contribution to $\sigma_{\pi N}$ and for $\sigma_{s}$ and $\sigma_{c}$ which are completely disconnected. With our increased statistics we find $\sigma_{\pi N}=36(2) \mathrm{MeV}, \sigma_{s}=37(8) \mathrm{MeV}$ and $\sigma_{c}=83(17) \mathrm{MeV}$. Comparison with other recent lattice QCD results and phenomenology is also shown in Fig. 3.

\section{First moments: $\langle x\rangle_{q},\langle x\rangle_{\Delta q},\langle x\rangle_{\delta q}$}

We consider the one derivative operators yielding the unpolarized moment $\langle x\rangle_{q}=\int_{0}^{1} d x x[q(x)+\bar{q}(x)]$, the helicity moment, $\langle x\rangle_{\Delta q}=\int_{0}^{1} d x x[\Delta q(x)-\Delta \bar{q}(x)]$ and the transversity moment $\langle x\rangle_{\delta q}=\int_{0}^{1} d x x[\delta q(x)+\delta \bar{q}(x)]$, where $q(x)=q(x)_{\downarrow}+q(x)_{\uparrow}, \Delta q(x)=q(x)_{\downarrow}-q(x)_{\uparrow}$ and $\delta q(x)=q(x)_{\perp}+q(x)_{\top}$.

In Fig. 4 we show the isovector unpolarized and transversity moments as the sink-source time separation is increased, as well as the results from the summation and the two-state fits. The quark momentum fraction given by $\langle x\rangle_{u-d}$ decreases as $t_{s}$ increases, as expected from previous studies, 


\begin{tabular}{ccccc}
\hline$g_{A}^{u-d}$ & $g_{A}^{u+d}$ (conn.) & $g_{A}^{u+d}$ (disconn.) & $g_{A}^{s}$ & $g_{A}^{c}$ \\
$1.21(3)(3)$ & $0.591(24)$ & $-0.15(2)$ & $-0.042(10)$ & $-0.0053(30)$ \\
\hline$g_{S}^{u-d}$ & $g_{S}^{u+d}($ conn. $)$ & $g_{S}^{u+d}$ (disconn.) & $g_{S}^{s}$ & $g_{S}^{c}$ \\
$0.93(25)(19)$ & $8.246(510)$ & $1.253(258)$ & $0.330(69)$ & $0.054(13)$ \\
\hline$g_{T}^{u-d}$ & $g_{T}^{u+d}($ conn. $)$ & $g_{T}^{u+d}$ (disconn.) & $g_{T}^{s}$ & \\
$1.004(21)(20)$ & $0.585(16)$ & $0.0007(11)$ & $-0.0004(6)$ & \\
\hline$\langle x\rangle_{u-d}$ & $\langle x\rangle_{u+d}$ (conn.) & $\langle x\rangle_{u+d+s}$ & $\langle x\rangle_{s}$ & \\
$0.194(9)(10)$ & $0.498(13)$ & $0.74(10)$ & $0.092(41)$ & \\
\hline$\langle x\rangle_{\Delta u-\Delta d}$ & $\langle x\rangle_{\Delta u+\Delta d}$ & $\langle x\rangle_{\Delta u+\Delta d}$ (disconn.) & $\langle x\rangle_{\Delta s}$ & \\
$0.259(9)(10)$ & $0.175(8)$ & $-0.011(24)$ & $-0.051(49)$ & \\
\hline$\langle x\rangle_{\delta u-\delta d}$ & $\langle x\rangle_{\delta u+\delta d}$ (conn.) & $\langle x\rangle_{\delta u+\delta d}$ (disconn.) & & \\
$0.273(17)(18)$ & $0.232(20)$ & $-0.0038(51)$ & & \\
\hline \multicolumn{5}{c}{}
\end{tabular}

Table 1: Values of the nucleon charges and first moments in the $\overline{\mathrm{MS}}$ scheme at $2 \mathrm{GeV}$ for the physical point ensemble. For the isovector quantities the first error is statistical and the second systematic determined by the difference between the values from the plateau and two-state fits.
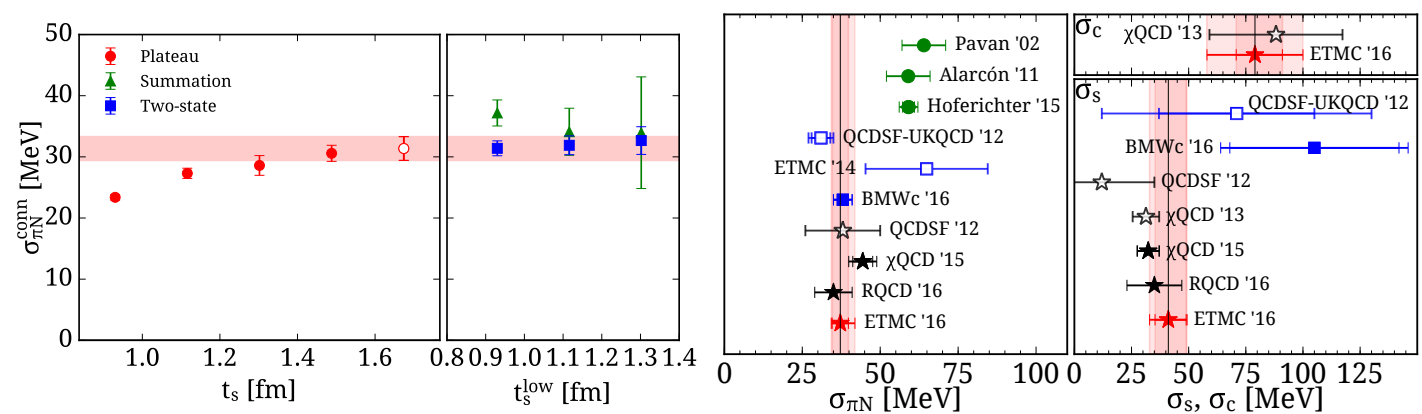

Figure 3: On the left we show the convergence of the connected contribution to $\sigma_{\pi N}$. On the right we show recent lattice QCD results on the light, strange and charm $\sigma$-terms.

approaching the experimental value. Similar behaviour is also observed for the polarized moment $\langle x\rangle_{\Delta u-\Delta d}$. The transversity for all three values of $t_{s}$ yields consistent results that also agree with the values extracted using two-state fits, pointing to less contamination due to excited states. Larger values of $t_{s}$ are under study. We have also computed the isoscalar quantities together with the disconnected contributions. These are given in Table I. For the isoscalar $\langle x\rangle_{u+d+s}$ we take into account perturbatively the mixing with the gluon momentum fraction $\langle x\rangle_{g}$. Neglecting the mixing we find $\langle x\rangle_{u+d+s}=0.81(11)$ and $\langle x\rangle_{u+d}=0.72(10)$.

\section{Gluon content of the nucleon}

Gluons can carry a significant amount of momentum and spin in the nucleon and thus far only a few studies have computed this contribution [7, 8]. In order to evaluate the gluon contribution to the nucleon spin we need to compute $J_{g}=\frac{1}{2}\left(A_{20}^{g}+B_{20}^{g}\right)$, that involves the momentum fraction $A_{20}^{g}=\langle x\rangle_{g}$ and $B_{20}^{g}$. As a first step we compute here the gluon momentum fraction from the nucleon matrix element of the gluon operator $O_{\mu \nu}=-G_{\mu \rho} G_{v \rho}$. The gluon momentum fraction is then extracted from $\left\langle N(0)\left|O_{44}-\frac{1}{3} O_{j j}\right| N(0)\right\rangle=m_{N}\langle x\rangle_{g}$.

The disconnected correlation function connecting a gluon loop is known to be very noisy. We thus employ several steps of stout smearing in order to remove the UV fluctuations in the gauge 

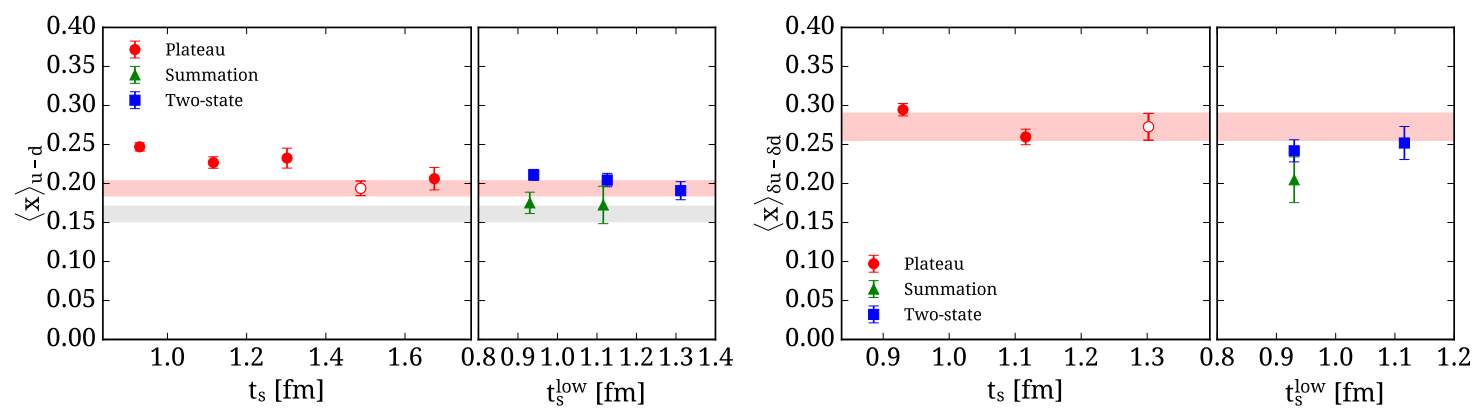

Figure 4: The isovector unpolarized (left) and helicity moments (right). The notation is the same as that of Fig. 1.

field. The methodology was tested for $N_{f}=2+1+1$ twisted mass fermions at $m_{\pi}=373 \mathrm{MeV}$ [8].

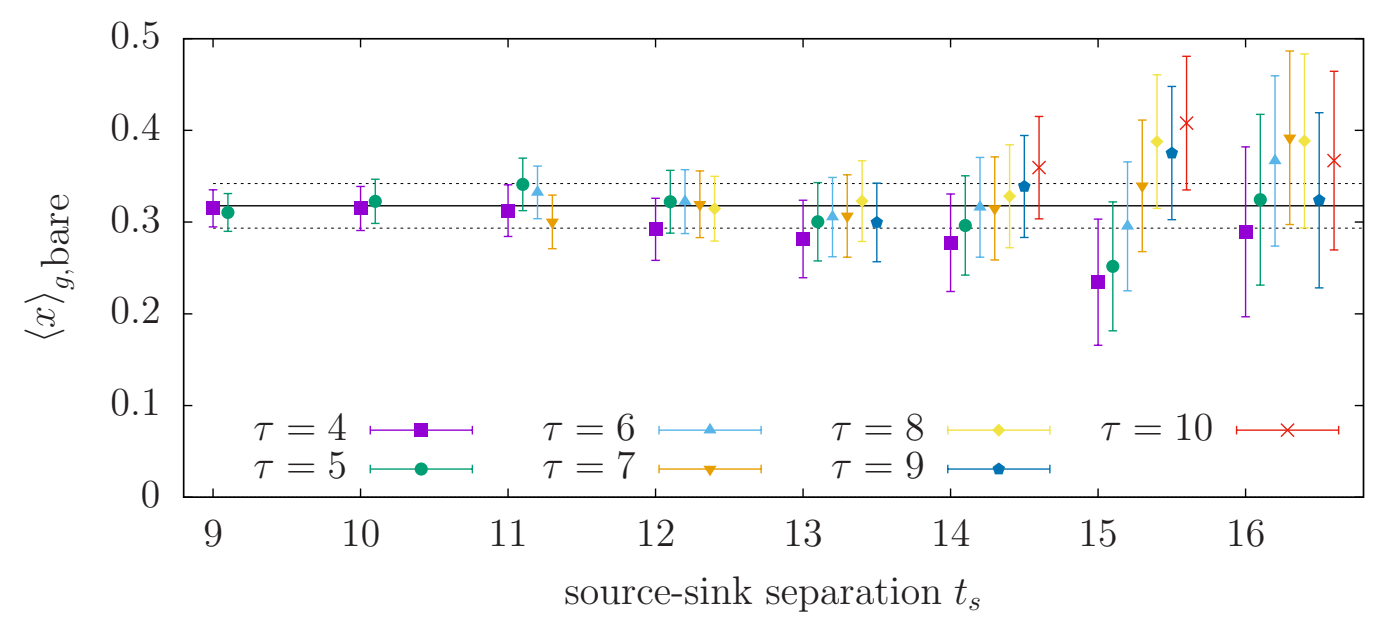

Figure 5: Gluon moment as a function of the sink-source time separation $t_{s}$ in units of the lattice spacing for various fits ranges $\tau$.

Using the physical ensemble and analyzing 2094 gauge configurations with 100 different source positions each, leading to over 200000 measurements, we obtain an accurate value for the bare gluon momentum fraction as shown in Fig. 5, namely $\langle x\rangle_{g \text {,bare }}=0.318(24)$. Due to mixing with the quark singlet operator, the renormalization and mixing coefficients have to be extracted from a one-loop perturbative lattice calculation. This yields $\langle x\rangle_{g}^{R}=Z_{g g}\langle x\rangle_{g}+Z_{g q}\langle x\rangle_{u+d+s}=$ $0.273(23)(24)$. The renormalization is carried out perturbatively using two-levels of stout smearing. The systematic error is the difference between using one- and two-levels of stout smearing. Having both the quark and gluon momentum fractions we can check whether the momentum sum is satisfied. We find: $\sum_{q}\langle x\rangle_{q}+\langle x\rangle_{g}=\langle x\rangle_{u+d}^{\text {conn }}+\langle x\rangle_{u+d+s}^{\text {disconn }}+\langle x\rangle_{g}=1.01(10)(2)$, which indeed is consistent with unity.

\section{Nucleon spin}

An analysis of the proton spin decomposition has been an important long-term goal for understanding the origin of spin in the nucleon, particularly after the the European Muon Collabora- 
tion [9] showed that the quark spin only contributes a surprisingly small amount to the total spin of the proton. Using our results on $g_{A}^{q}=\Delta \Sigma^{q}$ computed including disconnected contributions at the physical point we can compare directly to the experimental results.

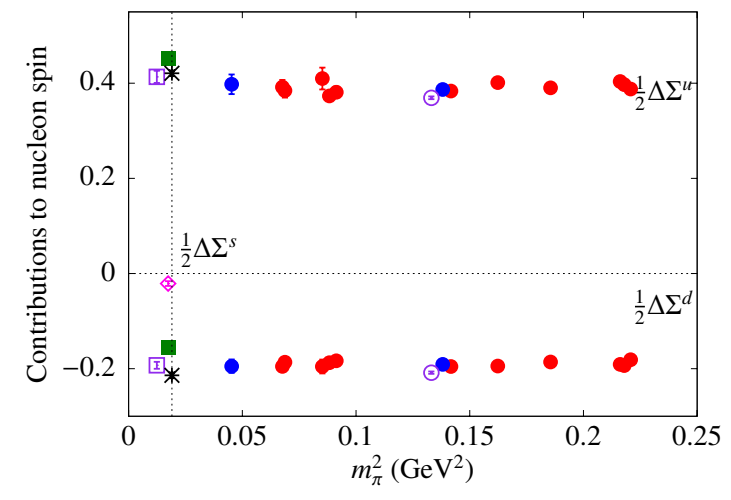

Figure 6: $\Delta \Sigma^{q}$ as a function of the pion mass using twisted mass fermions (TMF).
In Fig. 6 we show $\frac{1}{2} \Delta \Sigma^{q}$ for various $N_{f}=2$ (red circles) and $N_{f}=2+1+1$ (blue circles) TMF ensembles. The value for the physical ensemble is shown with the green squares and the experimental value with the asterisks. For the physical ensemble as well as for an $N_{f}=$ $2+1+1$ ensemble with $m_{\pi}=373 \mathrm{MeV}$ (B55) we include the effect of the disconnected contribution, which although small, shifts the values towards the experimental one.

The total quark and gluon spin $J^{q, g}=\frac{1}{2}\left(A_{20}^{q, g}(0)+B_{20}^{q, g}(0)\right)$ can be extracted from the nucleon matrix element of the one derivative vector operator that yields the generalized form factors $A_{20}(0)$ and $B_{20}(0)$. While $A_{20}$ can be extract directly at zero momentum transfer, $B_{20}$ must be extrapolated using results at finite momentum transfers. We found that disconnected contributions to $B_{20}(0)$ are consistent with zero and we therefore do not include them in this analysis. Disconnected contribution to $A_{20}$ are non-zero and are included for the physical and B55 ensembles. Using the spin sum rule $J^{q}=\left(\frac{1}{2} \Delta \Sigma^{q}+L^{q}\right)$ one can extract the orbital angular momentum $L^{q}$. The spin $J^{q}$ and $L^{q}$ are shown in Fig. 7 [10]. We note that the isovector and isoscalar momentum fraction are normalized with the same non-perturbatively determined renormalization constant neglecting the small mixing with the gluon momentum so that we can extract the individual quark contributions.
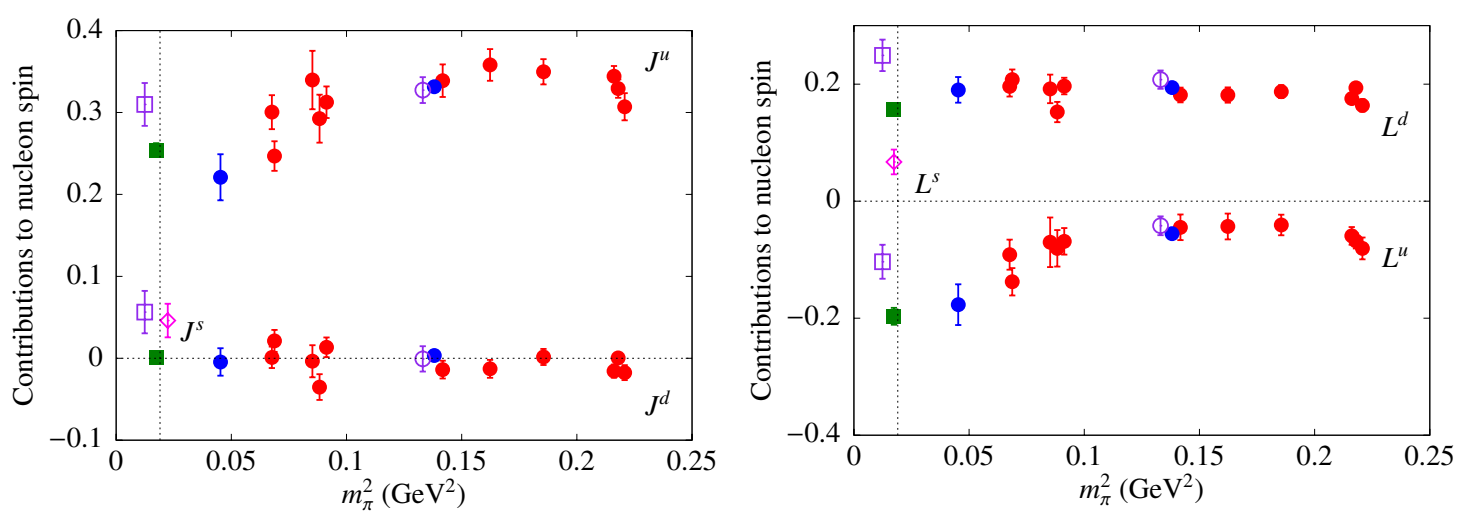

Figure 7: The total quark spin (left) and angular momentum $L^{q}$ (right) as a function of $m_{\pi}$. The notation is the same as in Fig. 6.

At the physical point we obtain:

$$
\frac{1}{2} \Delta \Sigma^{u}=0.413(13), \frac{1}{2} \Delta \Sigma^{d}=-0.193(7), \frac{1}{2} \Delta \Sigma^{s}=-0.021(5)
$$




$$
\begin{array}{rlr}
J^{u}=0.310(26), & J^{d}=0.056(26), & J^{s}=0.046(21) \\
L^{u}=-0.104(29), & L^{d}=0.249(27), & L^{s}=0.067(21)
\end{array}
$$

Using the spin sum rule $\frac{1}{2}=\sum_{q=u, d, s}\left(J^{q}\right)+J^{g}$ we deduce that $J^{g}=0.09(6)$, which is in agreement with the direct evaluation of the momentum fraction $\frac{1}{2}\langle x\rangle_{g}=0.136(12)$ [11]. This indicates that $B_{20}^{g}$ is small, which is in line with what is found for the quark connected contribution that yields $B_{20}^{u+d}=$ 0.012(20). Neglecting $B_{20}^{g}$, we find for the nucleon spin, $J_{N}=\sum_{q=u, d, s}\left(J^{q}\right)+J^{g}=0.51(5)(4)$, which satisfies the spin sum of $1 / 2$. The systematic error is the difference between neglecting i.e. using non-perturnbative renormalization and including perturbatively the mixing with $\langle x\rangle_{g}$.

\section{Conclusions}

Using high statistics we are able to compute both connected and disconnected contributions to the nucleon charges and to the first moment of parton distributions. Excited states contamination is carefully examined for all quantities. For the scalar charge and correspondingly the $\sigma$-terms the analysis is carried out for sink-source time separations up tot $1.7 \mathrm{fm}$ to ensure that the nucleon ground state dominates. For the axial charge, polarized and helicity moments larger sink-source time separations are under investigation. The quark spin decomposition shows that the intrinsic spin is in agreement with the experimental values, while we obtain non-zero disconnected contributions to the orbital angular momentum $L^{q}$.

Acknowledgments: We used computational resources on Piz Daint at the Swiss Supercomputing Center, under project ID s625 and s540, from the John von Neumann-Institute for Computing on the Juropa system and the BlueGene/Q system Juqueen at the research center in Jülich, from the Gauss Centre for Supercomputing on HazelHen (HLRS) and SuperMUC (LRZ), and on the Cy-Tera machine, The Cyprus Institute, funded by RPF, NEAYPODOMH/STPATH/0308/31. This project is partly supported by the EU Horizon 2020 research and innovation program Marie Sklodowska-Curie under grant agreement No 642069.

\section{References}

[1] J. R. Ellis, K. A. Olive and C. Savage, Phys. Rev. D 77, 065026 (2008) [arXiv:0801.3656 [hep-ph]].

[2] A. Abdel-Rehim et al. [ETM Collaboration], arXiv:1507.05068 [hep-lat].

[3] A. Abdel-Rehim et al., Phys. Rev. D 92, no. 11, 114513 (2015) Erratum: [Phys. Rev. D 93, no. 3, 039904 (2016)] [arXiv:1507.04936 [hep-lat]].

[4] A. Abdel-Rehim et al., PoS LATTICE 2014, 119 (2015) [arXiv:1411.6842 [hep-lat]].

[5] A. Abdel-Rehim et al. [ETM Collaboration], PoS (Lattice 2016) 155.

[6] A. Abdel-Rehim et al. [ETM Collaboration], Phys. Rev. Lett. 116, no. 25, 252001 (2016).

[7] Y. B. Yang et al., arXiv:1609.05937 [hep-ph].

[8] C. Alexandrou, V. Drach, K. Hadjiyiannakou, K. Jansen, B. Kostrzewa and C. Wiese, PoS LATTICE 2013, 289 (2014) [arXiv:1311.3174 [hep-lat]].

[9] J. Ashman et al. [European Muon Collaboration], Nucl. Phys. B 328, 1 (1989).

[10] C. Alexandrou et al., PoS DIS 2016, 240 (2016) [arXiv:1609.00253 [hep-lat]].

[11] C. Alexandrou, M. Constantinou, K. Hadjiyiannakou, K. Jansen, H. Panagopoulos and C. Wiese, arXiv:1611.06901 [hep-lat]. 